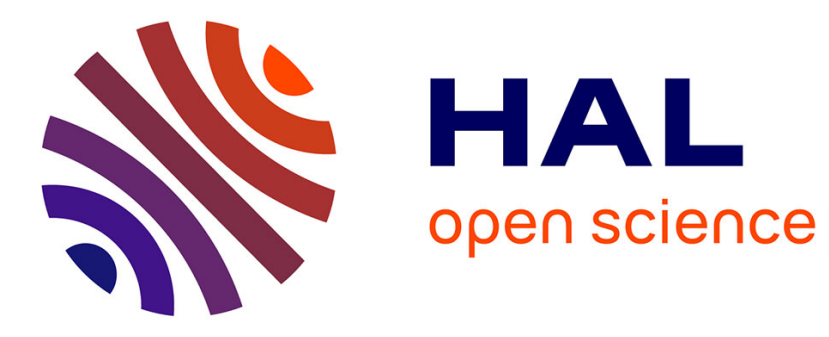

\title{
Euler-Bernoulli beam flatness based control with constraints
}

Maria Bekcheva, Luca Greco, Hugues Mounier, Alban Quadrat

\section{To cite this version:}

Maria Bekcheva, Luca Greco, Hugues Mounier, Alban Quadrat. Euler-Bernoulli beam flatness based control with constraints. IEEE 9th International Workshop on Multidimensional (nD) Systems, Sep 2015, Vila Real, Portugal. pp.1-6, 10.1109/NDS.2015.7332635 . hal-01264912

\section{HAL Id: hal-01264912 \\ https://hal-centralesupelec.archives-ouvertes.fr/hal-01264912}

Submitted on 2 Feb 2016

HAL is a multi-disciplinary open access archive for the deposit and dissemination of scientific research documents, whether they are published or not. The documents may come from teaching and research institutions in France or abroad, or from public or private research centers.
L'archive ouverte pluridisciplinaire HAL, est destinée au dépôt et à la diffusion de documents scientifiques de niveau recherche, publiés ou non, émanant des établissements d'enseignement et de recherche français ou étrangers, des laboratoires publics ou privés. 


\title{
Euler-Bernoulli beam flatness based control with constraints
}

\author{
Maria Bekcheva*, Luca Greco*, Hugues Mounier*, Alban Quadrat* \\ ${ }^{*}$ Laboratoire des Signaux et Systèmes \\ CentraleSupélec, 3, rue Joliot Curie, \\ 91192 Gif sur Yvette, FRANCE \\ Email: \{maria.bekcheva,luca.greco,hugues.mounier,alban.quadrat\}@1ss.supelec.fr
}

\begin{abstract}
The control of infinite dimensional systems with constraints is a notoriously difficult task. We consider a general class of linear systems governed by partial differential equations with boundary control. This problem is here treated in a quite natural manner through the freeness property, the analogue of differential flatness for linear systems. Any variable is then expressed as infinite order differential operators applied to the basis components, the analogue of the flat output components. The specialisation of the basis components are functions which are both of Gevrey regularity (in order for the infinite order differential operators to be convergent) and pertaining the flexibility of polynomial splines. An illustration is made through an Euler Bernoulli beam example.
\end{abstract}

Index Terms-Flexible structures, infinite dimensional systems, rings, modules, controllability, Euler-Bernoulli partial differential equation, Gevrey-Roumieu functions, input constraints.

\section{INTRODUCTION}

Control of infinite dimensional systems with constraints is known to be a difficult task. We here consider the case of systems giverned by partial differential equations with a control at the boundary. We shall study the class of free systems, the linear analogue of nonlinear finite dimensional differentially flat systems, and make use of algebraic techniques. The differential flatness (see [17]) gives a solution to systems governed by ordinary differential equations. As noticed in [17], this method is not restricted to ordinary differential equations and can be adapted to delay differential systems and partial differential equations with boundary control.

The freeness of a certain modules, whose properties are obtained through homological arguments (see, e.g., [11], [29]; We especially use the resolution of Serre's conjecture by Quillen [46] and Suslin [56], already exploited in [39]; see also [18], [19], [13], [45]), enables, by assigning a trajectory to a basis of this module, to obtain the desired trajectory tracking.

We shall, as systematized in [53], envision the equations of the system as a Cauchy problem in the spatial variable, a problem which is well posed in some suitable spaces of generalized functions: the desired convolutional system is obtained by first solving the Cauchy problem and plugging its solution into the boundary conditions, i.e., the equations imposed by the boundary conditions further restrict the Cauchy data.

Differentially flat systems with constraints are generally attacked through the use of optimal control problems (see, e.g. [14], [15], [43], [44], [55]). We here propose to embed the constraint fulfillment in the trajectory design. We thus specialize the basis (or flat output) to the convolution of a so called Gevrey function with a polynomial B-spline.

In Section II we briefly recall general definitions about $R$ linear systems. In the next Section, bounday value problems are modeled as modules over a ring parametrized by space. In Section IV various controllability definitions are recalled. Section V is the main section of the paper, dealing with constraints fulfillment. Application to an Euler-Bernoulli beam is the subject of Section VI.

\section{II. $R$-LINEAR SYSTEMS}

We shall consider in this section quite general definitions for linear systems viewed as modules over a ring. In the next section, we shall be more specific in order to describe boundary value problems as modules over a ring parametrized by space.

Definition 1: An $R$-system $\Lambda$, or a system over $R$, is an $R$-module. A presentation matrix of a finitely presented $R$ system $\Sigma$ is a matrix $P$ such that $\Sigma \cong[v] /[P v]$ where $[v]$ is free with basis $v$. An output $\boldsymbol{y}$ is a subset, which may be empty, of $\Lambda$. An input-output R-system, or an input-output system over $R$, is an $R$-dynamics equipped with an output.

The next definition allows, by extension of scalars, to obtain much nicer algebraic properties when needed.

Definition 2: Let $A$ be an $R$-algebra and $\Lambda$ be an $R$-system. The $A$-module $A \otimes_{R} \Lambda$ is an $A$-system, which extends $\Lambda$.

\section{BOUNDARY VALUE PROBLEMS AS SYSTEMS PARAMETRIZED BY SPACE}

We shall here consider boundary value PDE systems as modules over rings. A space parametrization is embedded in the chosen rings.

\section{A. Model class}

Models are here considered as space dynamics with time differential operator coefficients. 
1) Distributed equations: The envisioned model equations are based on a Cauchy-Kowalevski form:

$$
\begin{array}{r}
\partial_{x} \boldsymbol{w}_{i}=A_{i} \boldsymbol{w}_{i}+B_{i} \boldsymbol{u}, \quad \boldsymbol{w}_{i}: \Omega_{i} \rightarrow\left(\mathcal{D}^{\prime *}\right)^{p}, \\
\boldsymbol{u} \in\left(\mathcal{D}^{\prime *}\right)^{m}, \quad A_{i} \in\left(\mathbb{R}\left[\partial_{t}\right]\right)^{p_{i} \times p_{i}}, \quad B_{i} \in\left(\mathbb{R}\left[\partial_{t}\right]\right)^{p_{i} \times m}, \\
i \in\{1, \ldots, l\}
\end{array}
$$

where $\boldsymbol{w}_{1}, \ldots, \boldsymbol{w}_{l}$ are the distributed variables, $\boldsymbol{u}=$ $\left(u_{1}, \ldots, u_{m}\right)$ the lumped variables, and $\mathcal{D}^{\prime} *$ denotes a space of (ultra -) distributions.

2) Assumptions: We shall make two assumptions:

- The intervals $\Omega_{1}, \ldots, \Omega_{l}$ are given by an open neighborhood of

$$
\begin{gathered}
\tilde{\Omega}_{i}=\left[x_{i, 0}, x_{i, 1}\right], \quad \ell_{i}=x_{i, 1}-x_{i, 0}=q_{i} \ell \\
q_{i} \in \mathbb{Q}, \ell \in \mathbb{R}
\end{gathered}
$$

Without loss of generality, assume $x_{i, 0}=0$.

- The characteristic polynomials of the matrices $A_{1}, \ldots, A_{l}$ can be written

$$
\begin{aligned}
P_{i}(\lambda) & :=\operatorname{det}\left(\lambda I-A_{i}\right)=\sum_{\nu=0}^{p_{i}} a_{i, \nu} \lambda^{\nu}, \\
a_{i, \nu} & =\sum_{\nu+\mu \leq p_{i}} a_{i, \nu, \mu} \partial_{t}^{\mu}
\end{aligned}
$$

with $a_{i, j, k} \in \mathbb{R}, a_{i, p_{i}, 0}=1$. Moreover, their principal parts $\sum_{\mu+\nu=p_{i}} a_{i, \mu, \nu} \partial_{t}^{\mu} \lambda^{\nu}$ are hyperbolic w.r.t. the time $t$, i.e. the roots of $\sum_{\mu+\nu=p_{i}} a_{i, \mu, \nu} \lambda^{j}$ are real.

Remark 1. - Note that the above assumptions apply to most spatially one-dimensional boundary controlled evolution equations including Euler-Bernoulli or Timoshenko beam equations, more general parabolic diffusion-reaction-convection equations, damped and undamped wave-equations, etc. The only notable exception is the case where the maximal order derivative is a mixed one, such as, e.g. models of structural damping $\left(\alpha \partial_{t}+1\right)\left(\partial_{x}^{2}-\partial_{t}^{2}\right) \boldsymbol{w}=0$

3) Boundary conditions: The models are completed by the following boundary conditions

$$
\sum_{i=1}^{l} L_{i} \boldsymbol{w}_{i}(0)+R_{i} \boldsymbol{w}_{i}\left(\ell_{i}\right)+D \boldsymbol{u}=0
$$

with $D \in\left(\mathbb{R}\left[\partial_{t}\right]\right)^{q \times m}$ and $L_{i}, R_{i} \in\left(\mathbb{R}\left[\partial_{t}\right]\right)^{q \times p_{i}}$.

\section{B. Solution of the Cauchy Problem}

Some properties of the solution of the Cauchy problem (1a) with initial conditions given by $x=\xi$, i.e.

$$
\partial_{x} \boldsymbol{w}=A \boldsymbol{w}+B \boldsymbol{u}, \quad \boldsymbol{w}(\xi)=\boldsymbol{w}_{\xi}
$$

with $A \in\left(\mathbb{R}\left[\partial_{t}\right]\right)^{p \times p}, B \in\left(\mathbb{R}\left[\partial_{t}\right]\right)^{p \times q}$ as assumed in the previous section for $A_{i}, B_{i}$, will be used. The notation of the previous section is used in what follows, dropping the index $i \in\{1, \ldots, l\}$.

Consider the initial value problem

$P\left(\partial_{x}\right) v(x)=0, \quad\left(\partial_{x}^{j} v\right)(0)=v_{j} \in \mathcal{E}^{*}(\mathbb{R}), \quad j=0, \ldots, p-1$ associated with the characteristic equation

$$
P(\lambda):=\operatorname{det}(\lambda I-A)=\sum_{j=0}^{p} a_{j} \lambda^{j}, \quad a_{j}=\sum_{j+\mu \leq p} a_{j, \mu} \partial^{\mu} .
$$

According to $[24$, Thrm. 12.5.6] or [49, Thrm 2.5.2,Prop. 2.5.6] the initial value problem (3) has a unique solution which may be written as

$$
v(x)=\sum_{j=0}^{p-1} C_{j}(x) v_{j}
$$

where juxtaposition of symbols means convolution and $C_{0}, \ldots, C_{p-1}$ are smooth functions mapping $\Omega$ to the space of compactly supported Beurling ultradistributions $\mathcal{E}^{*}(\mathbb{R}):=$ $\mathcal{E}^{\prime}(p /(p-1))(\mathbb{R})$ of Gevrey order $p /(p-1)$. The functions $C_{0}, \ldots, C_{p-1}$ satisfy $(k, j \in\{0, \ldots, p-1\})$

$$
\partial_{x}^{k} C_{j}(0)= \begin{cases}1, & k=j \\ 0, & k \neq j\end{cases}
$$

and

$$
\begin{aligned}
& \partial_{x} C_{j}=C_{j-1}-a_{j} C_{p-1}, \quad j=1, \ldots, p-1, \\
& \partial_{x} C_{0}=-a_{0} C_{p-1} .
\end{aligned}
$$

With this preparatory steps, the unique solution $x \mapsto \Phi(x, \xi)$ of the initial value problem (2) can be expressed as

$$
\boldsymbol{w}(x)=\Phi(x, \xi) \boldsymbol{w}_{\xi}+\Psi(x, \xi) \boldsymbol{u}
$$

Therein, $\Phi(x, \xi) \in \mathcal{E}^{\prime *}(\mathbb{R})^{p \times p}$ is the fundamental matrix of the initial value problem

$$
\boldsymbol{w}(x)=\Phi(x, \xi) \boldsymbol{w}(x), \quad \boldsymbol{w}(\xi)=\boldsymbol{w}_{\xi}
$$

and $\Psi(x, \xi) \in \mathcal{E}^{*}(\mathbb{R})^{p \times m}$ corresponds to the particular solution of (2) with vanishing data $w_{\xi}=0$.

Explicit expressions for $\Phi$ and $\Psi$ can be given using the ultradistribution-valued functions $C_{0}, \ldots, C_{p-1}$

$$
\Phi(x, \xi)=\sum_{j=0}^{p-1} A^{j} C_{j}(x-\xi), \quad \Psi(x, \xi)=\int_{\xi}^{x} \Phi(x, \zeta) d \zeta B .
$$

Substituting the general solutions of the initial value problems into the boundary conditions, one obtains the following linear system of equations:

$$
\boldsymbol{w}(x)=W_{\boldsymbol{\xi}}(x) \boldsymbol{c}_{\boldsymbol{\xi}}, \quad P_{\boldsymbol{\xi}} c_{\boldsymbol{\xi}}=0 .
$$

Here, $\boldsymbol{\xi}=\left(\xi_{1}, \ldots, \xi_{n}\right), \boldsymbol{c}_{\boldsymbol{\xi}}^{T}=\left(\boldsymbol{w}_{1}^{T}\left(\xi_{1}\right) \cdots \boldsymbol{w}_{l}^{T}\left(\xi_{l}\right), \boldsymbol{u}^{T}\right)$,

$$
\begin{gathered}
W_{\boldsymbol{\xi}}=\left(\begin{array}{cccc}
\Phi_{1}\left(x, \xi_{1}\right) & 0 & 0 & \Psi_{1}\left(x, \xi_{1}\right) \\
0 & \ddots & 0 & \vdots \\
0 & \cdots & \Phi_{l}\left(x, \xi_{l}\right) & \Psi_{l}\left(x, \xi_{l}\right)
\end{array}\right) \\
P_{\boldsymbol{\xi}}=\left(P_{\boldsymbol{\xi}, 1} \cdots P_{\boldsymbol{\xi}, l+1}\right)
\end{gathered}
$$


with

$$
\begin{aligned}
P_{\boldsymbol{\xi}, i} & =L_{i} \Phi_{i}\left(0, \xi_{i}\right)+R_{i} \Phi_{i}\left(\ell_{i}, \xi_{i}\right), \quad i=1, \ldots, l \\
P_{\boldsymbol{\xi}, l+1} & =D+\sum_{i=1}^{l} L_{i} \Psi_{i}\left(0, \xi_{i}\right)+R_{i} \Psi_{i}\left(\ell_{i}, \xi_{i}\right) .
\end{aligned}
$$

A possible choice for the coefficient ring is the ring $\mathcal{R}_{\mathbb{R}}^{I}\left[s, \mathfrak{S}, \mathfrak{S}^{I}\right]$. Here, for any $\mathbb{X} \subseteq \mathbb{R}, \mathcal{R}_{\mathbb{X}}^{I}=\left[\mathfrak{S}_{\mathbb{X}}, \mathfrak{S}_{\mathbb{X}}^{I}\right]$, with

$$
\begin{aligned}
\mathfrak{S} & =\{C, S\}, & \mathfrak{S}_{\mathbb{X}} & =\{C(z \ell), S(z \ell) \mid z \in \mathbb{X}\}, \\
\mathfrak{S}^{I} & =\left\{C^{I}, S^{I}\right\}, & \mathfrak{S}_{\mathbb{X}}^{I} & =\left\{C^{I}(z \ell), S^{I}(z \ell) \mid z \in \mathbb{X}\right\},
\end{aligned}
$$

$\ell$ defined as in $(1 b)$, and

$$
S^{I}(x)=\int_{0}^{x} S(\zeta) d \zeta, \quad C^{I}(x)=\int_{0}^{x} C(\zeta) d \zeta .
$$

Inspired by the results given in [38], [2], [22], and in view of the simplification of the analysis of the module properties, instead of the ring $\mathcal{R}_{\mathbb{R}}^{I}$, we shall use a slightly larger ring, given by $\mathcal{R}_{\mathbb{R}}=\mathbb{C}(s)\left[\mathfrak{S}_{\mathbb{R}}\right] \cap \mathcal{E}^{\prime *}$.

Definition 3: The convolutional system $\Sigma$ associated with the boundary value problem (1) is the module generated by the elements of $c_{\xi}$ over $\mathcal{R}=\mathcal{R}_{\mathbb{R}}\left[\mathfrak{S}, \mathfrak{S}^{I}\right]$ with presentation matrix $P_{\boldsymbol{\xi}}$. By $\Sigma_{\mathbb{R}}\left(\right.$ resp. $\left.\Sigma_{\mathbb{Q}}\right)$ we denote the same system but viewed as a module over $\mathcal{R}_{\mathbb{R}}\left(\right.$ resp. $\left.\mathcal{R}_{\mathbb{Q}}\right)$.

\section{SySTEM CONTROLLABILITIES}

\section{A. General controllabilities}

In this section we emphasize several controllability notions which are defined directly without referring to a solution space. Let us start with some purely algebraic definitions:

Definition 4 (see, e.g. , [20, Def. 2.4.]): Let $A$ be an $R$ algebra. An $R$-system $\Lambda$ is said to be $A$-torsion free controllable (resp. A-projective controllable, A-free controllable) if the $A$-module $A \otimes_{R} \Lambda$ is torsion free (resp. projective, free). An $R$-torsion free (resp. R-projective, $R$-free) controllable $R$ system is simply called torsion free (resp. projective, free) controllable.

Elementary homological algebra (see, e.g., [50]) yields

Proposition 1: $A$-free (resp. A-projective) controllability implies $A$-projective (resp. $A$-torsion free) controllability.

Proposition 2: $R$-free controllability implies $A$-free controllability for any $R$-algebra $A$. More generally, given any $R$ system $\Sigma$ that is a direct sum of a torsion module $\mathrm{t} \Sigma$ and a free module $\Lambda$, the extended system $A \otimes_{R} \Sigma$ is a direct sum of the torsion module $A \otimes_{R} \mathrm{t} \Sigma$ and the free module $A \otimes_{R} \Lambda$.

The importance of the notions of torsion free and free controllability is intuitively clear: While the first one refers to the absence of a nontrivial subsystem which is governed by an autonomous system of equations, the latter refers to the possibility to freely express all system variables in terms of a basis of the system module. For this reason, and, secondarily, in reminiscence to the theory of nonlinear finite dimensional systems, we have the following:

Definition 5: Take an $A$-free controllable $R$-system $\Lambda$ with a finite output $y$. This output is said to be A-flat, or A-basic, if $\boldsymbol{y}$ is a basis of $A \otimes_{R} \Lambda$. If $A \cong R$ then $\boldsymbol{y}$ is simply called flat, or basic.

\section{CONTROL With CONSTRAINTS}

\section{A. Gevrey functions}

For an $A$-free controllable $R$-system $\Lambda$, the basis $\boldsymbol{y}$ is introduced in order to express all system variables through infinite order differential operators:

$$
\begin{aligned}
\boldsymbol{w}(x, t) & =\sum_{i=0}^{\infty} a_{i}(x) y^{(i)}(t) \\
\boldsymbol{u}(t) & =\sum_{i=0}^{\infty} b_{i} y^{(i)}(t)
\end{aligned}
$$

This representation make sense only if the series (10a) and (10b) can be made convergent. When an appropriate basis $y$ is chosen, these series lead in particular to an open loop control.

Definition 6 (Gevrey Class): [see, e.g., [25], [48], [49]] A smooth function $\phi: \mathbb{R} \rightarrow \mathbb{R}$ is of Gevrey order $\alpha$ if on any compact subset $K \subset \mathbb{R}$

$$
\exists m_{K}, \gamma_{K} \in \mathbb{R}^{+}, \forall k \in \mathbb{N}, \quad \sup _{t \in K}\left|\phi^{(k)}(t)\right| \leq \frac{m_{K}}{\gamma_{K}^{k}}(k !)^{\alpha} .
$$

The functions of Gevrey order $\alpha<1$ are entire, while analytic for $\alpha=1$ and non-analytic if $\alpha>1$.

The Taylor expansion of a smooth function is not convergent, unless the function is analytic. The Gevrey order $\alpha$ estimates this divergence. Gevrey functions of order $\alpha>1$ have divergent Taylor expansion; the larger $\alpha$, the more divergent the Taylor expansion. Important properties of analytic functions generalize to Gevrey functions of order $\alpha>1$ : the scaling, addition, multiplication and derivation of Gevrey functions of order $\alpha \leq 1$ is of order $\alpha$. But contrary to analytic functions, functions of order $\alpha>1$ may be constant on an open set without being constant everywhere.

\section{B. Identity approximation}

We shall make use of identity approximation whose definition we recall:

Definition 7: An identity approximation is a family $\phi_{\varepsilon}$ in $L_{1}(\mathbb{R})$ indexed by $\varepsilon>0$ such that:

1) $\left\|\phi_{\varepsilon}\right\|_{1}$ is bounded, independently of $\varepsilon>0$.

2) $\forall \varepsilon>0, \quad \int_{\mathbb{R}} \phi_{\varepsilon}=1$.

3) $\forall \eta>0, \quad \lim _{\varepsilon \rightarrow 0} \int_{|t|<\eta}\left|\phi_{\varepsilon}\right|=0$.

The following regularisation result explains the previous terminology

Proposition 3: Consider an identity approximation $\left(\phi_{\varepsilon}\right)$. For any function $f$ in $L_{p}(1 \leqslant p<\infty)$ the sequence $\left(\phi_{\varepsilon} * f\right)$ converges towards $f$ in $L_{p}$.

Thus, the sequence $\left(\phi_{\varepsilon}\right)$ can be seen to converge to the Dirac distribution. The following lemma is a useful construction of an identity approximation.

Lemma 1: Let $\phi: \mathbb{R} \rightarrow \mathbb{C}$ be a function in $\mathcal{D}(\mathbb{R})$ with non zero integral, then the sequence $\left(\phi_{\varepsilon}\right)$ with

$$
\phi_{\varepsilon}(t)=\frac{\phi(t / \varepsilon)}{\varepsilon \int_{\mathbb{R}} \phi(\tau) d \tau}
$$


is an identity approximation.

1) Gevrey identity approximation: Consider the following function

$$
g(\tau)= \begin{cases}\exp \left(-\left(1-\tau^{2}\right)^{-\sigma}\right), & \text { if } \tau \in[-1,1] \\ 0, & \text { otherwise }\end{cases}
$$

which is Gevrey of order $1+1 / \sigma$ and with compact support $[-1,1]$.

The previous construction leads to an identity approximation which is Gevrey of order $1+1 / \sigma$ (see [35], [53]:

$$
g_{\varepsilon}(t)=\frac{g(t / \varepsilon)}{\varepsilon \int_{\mathbb{R}} g(\tau) d \tau}
$$

\section{Basis as a B-spline Gevrey approximation}

We shall consider the following functions for the basis $y$ :

$$
y=g_{\varepsilon} * S
$$

where $\mathrm{S}$ is a polynomial B-spline curve of order $m$ (see [8]) and $g_{\varepsilon}$ is the previously defined Gevrey identity approximation.

Since $y$ is a convolution with the Gevrey function $g_{\varepsilon}$ it is Gevrey of the same order as $g_{\varepsilon}$. Since $g_{\varepsilon}$ is an identity approximation:

$$
\lim _{\varepsilon \rightarrow 0} g_{\varepsilon} * S=S
$$

Hence, to design a reference trajectory $y_{r}$ for $y$, one has the same flexibility as in the B-spline curve design.

The variables $\boldsymbol{w}(x, t)$ and $\boldsymbol{u}(t)$ are thus expressed as

$$
\begin{aligned}
\boldsymbol{w}(x, t) & =\left(\sum_{i=0}^{\infty} a_{i}(x) g_{\varepsilon}^{(i)}(t)\right) * S(t) \triangleq A_{\varepsilon}(x, t) * S(t) \\
\boldsymbol{u}(t) & =\left(\sum_{i=0}^{\infty} b_{i} g_{\varepsilon}^{(i)}(t)\right) * S(t) \triangleq B_{\varepsilon}(t) * S(t)
\end{aligned}
$$

in virtue of the identity $\dot{f} * g=f * \dot{g}$.

\section{Constraints fulfillment}

By Young's inequality, one obtains

$$
\|\boldsymbol{u}\|_{1}=\left\|B_{\varepsilon} * S\right\|_{1} \leqslant\left\|B_{\varepsilon}\right\|_{1}\|S\|_{1}
$$

Since $g_{\varepsilon}$ is Gevrey of order $1+1 / \sigma$, one has the following estimates for its derivatives:

$$
\left\|g_{\varepsilon}^{(i)}\right\|_{\infty} \leqslant \frac{m_{I}}{\gamma_{K}^{i}}(i !)^{1+\frac{1}{\sigma}}
$$

Considering the preceding inequality for $i=0$, one gets this possible choice $m_{I}$ :

$$
m_{I}=\left\|g_{\varepsilon}\right\|_{1}=g_{\varepsilon}(0)=\frac{1}{e \varepsilon I_{g}}
$$

where $I_{g}=\int_{\mathbb{R}} g(\tau) d \tau$.
Then, for $i=1$, one obtains a possible $\gamma_{K}$

$$
\gamma_{K}=\frac{\left\|g_{\varepsilon}\right\|_{\infty}}{\left\|\dot{g}_{\varepsilon}\right\|_{\infty}}=-\frac{\left(1-t^{* 2}\right)^{\sigma+1}}{2 \sigma t^{*} g\left(t^{*}\right) e \varepsilon I_{g}}
$$

Note that $t^{*} \in[-\varepsilon, 0]$ and

$$
\forall i \in I, \quad \dot{g}\left(t^{*}\right)>\dot{g}\left(t_{i}\right) .
$$

Then, the $L_{1}$ norm for $B_{\varepsilon}$ can be estimated as

$$
\begin{aligned}
\left\|B_{\varepsilon}\right\|_{1} & =\left\|\sum_{i=0}^{\infty} b_{i} g_{\varepsilon}^{(i)}(t)\right\|_{1} \leqslant \sum_{i=0}^{\infty}\left\|b_{i} g_{\varepsilon}^{(i)}(t)\right\|_{1} \\
& =\sum_{i=0}^{\infty}\left|b_{i}\right|\left\|g_{\varepsilon}^{(i)}(t)\right\|_{1} \leqslant \sum_{i=0}^{\infty} 2 \varepsilon\left|b_{i}\right|\left\|g_{\varepsilon}^{(i)}(t)\right\|_{\infty} \\
& \leqslant \sum_{i=0}^{\infty} 2 \varepsilon\left|b_{i}\right| \frac{m_{I}}{\gamma_{K}^{i}}(i !)^{1+\frac{1}{\sigma}}
\end{aligned}
$$

Then, $\|S\|_{1}$ is estimated since an approximating B-spline curve is always contained in the convex hull defined by its control points. Thus, one has to choose the highest control point of $S$ such that

$$
\|\boldsymbol{u}\|_{1}=\left\|B_{\varepsilon} * S\right\|_{1} \leqslant\left\|B_{\varepsilon}\right\|_{1}\|S\|_{1}
$$

is bounded by the constraint on the control.

Remark 2. - In practice, the infinite series (13) will be truncated (for implementation reasons) to a sufficiently high order. Since the bound we will consider in (15) is of uniform type (i.e. we take norms inside the sum), the inequality (14) will still be fulfilled.

\section{Vi. Application to An Euler-Bernoulli BEAM}

\section{A. The model}

The model of an Euler-Bernoulli beam is described below (see [1]).

$$
\begin{aligned}
\frac{\partial^{2} w}{\partial t^{2}} & =-\frac{\partial^{4} w}{\partial^{4} x} \\
w(0, t) & =0, \quad \frac{\partial w(0, t)}{\partial x}=L u(t) \\
\frac{\partial^{2} w(1, t)}{\partial x^{2}} & =-\lambda \frac{\partial^{3} w}{\partial x^{2} \partial t}(1, t), \\
\frac{\partial^{3} w(1, t)}{\partial x^{3}} & =\mu \frac{\partial^{2} w}{\partial t^{2}}(1, t)
\end{aligned}
$$

with

$$
\lambda=\frac{J}{\rho S L^{3}}, \quad \mu=\frac{M}{\rho S L}
$$

\section{B. Open loop control}

It has been shown in [1] that the system corresponding to eqs. (17) is free over a suitable ring.

The state and input parametrizations can be determined as:

If $y(t)$ is of class Gevrey, with $\alpha<2$, the following series, which corresponds to $\cosh (\sqrt{2 s}) y$ is absolutely convergent

$$
\sum_{n \geq 0} \frac{2^{n}}{(2 n) !} y^{(n)}(t)
$$


For all specialisation of the basis $y$ to a function of the class Gevrey, $\alpha<2$, equation (??) yields

$$
\begin{aligned}
u(t)= & \frac{-J_{m}}{L \alpha^{2}}\left[1+\sum_{n=0}^{\infty} \frac{2^{2 n+1}}{(4 n+4) !}\left((1+\lambda \mu) \frac{d^{2}}{d t^{2}}+\right.\right. \\
& \left.\left.(4 n+4)\left(\mu+\frac{4 n+3}{2} \lambda\right)\right) \frac{d^{2 n+4}}{d t^{2 n+4}}\right] y(\tau)+ \\
& \frac{E I}{L^{2}}\left[\sum _ { n = 0 } ^ { \infty } \frac { 2 ^ { 2 n + 1 } } { ( 4 n + 4 ) ! } \left((4 n+4)\left(\frac{1}{2}+\frac{\lambda \mu}{2}\right) \frac{d^{2}}{d t^{2}}+\right.\right. \\
& \left.\left.(4 n+3)\left(\mu+\frac{(4 n+1)(4 n+2)}{2} \lambda\right)\right) \frac{d^{2 n+2}}{d t^{2 n+2}}\right] y(t)
\end{aligned}
$$

and with (??),

$$
\begin{aligned}
& w(x, t)= {\left[\sum _ { n = 0 } ^ { \infty } \frac { ( - 1 ) ^ { n } s ^ { 2 n } } { ( 4 n ) ! } \left(\frac{x^{4 n+1}}{2(4 n+1)}+\right.\right.} \\
&\left.\left.\frac{(\Im-}{\Re)(1+i-x)^{4 n+1}}+\mu \Im(1+i-x)^{4 n}\right)\right] y(t)+ \\
& {\left[\sum _ { n = 0 } ^ { \infty } \frac { ( - 1 ) ^ { n } s ^ { 2 n + 2 } } { ( 4 n + 4 ) ! } \left(\frac{\lambda \mu}{2}+\right.\right.} \\
& \frac{(4 n+2) !}{(4 n+4) !}\left[(\Im-\Re)(1+i-x)^{4 n+1}-x^{4 n+1}\right] \\
&\left.\left.-\lambda(4 n+3)(4 n+4) \Re(1+i-x)^{4 n+2}\right)\right] y(t)
\end{aligned}
$$

where $\Re$ (resp. $\Im$ ) denotes the real (resp. imaginary) part. In other words, the two relations above define a family of trajectories for the hybrid system (17).

Then, the constraint depicted in (16) can be satisfied when replacing the $b_{i}$ 's by the ones found in the expression above.

\section{CONCLUSION}

We have outlined a framework for tackling boundary controlled flat PDE systems open loop trajectory tracking with contraints. We have made use of Gevrey identity approximation convolved with a polynomial B-spline for the basis. This retains the flexibility of approximating polynomial B-splines while maintaining the Gevrey character. Constraints fulfillment is ensured through young's inequality and Gevrey estimates. An application to the Euler-Bernoulli beam is outlined.

\section{REFERENCES}

[1] Y. Aoustin, M. Fliess, H. Mounier, P. Rouchon and J. RudolPh, Theory and practice in the motion planning control of a flexible robot arm using Mikusiǹski operators, Proc. of the Fifth IFAC Symposium on Robot Control, , 2, 1997, pp. 287-293.

[2] D. Brethé and J.J. Loiseau, A result that could bear fruit for the control of delay-differential systems. Proc. 4th IEEE Mediterranean Symp. Control Automation, Chania, Greece, 1996, pp. 168-172.

[3] J. Brewer, J. BunCE and F. V. VLECK, Linear Systems over Commutative Rings, Marcel Dekker, New York, 1986.

[4] D. Buchsbaum and D. Eisenbud, What makes a complex exact?, $J$. Algebra, 25, 1973, pp. 259-268.

[5] S.Y. Chung And J. Chung, There exist no gaps between Gevrey differentiable and nowhere Gevrey differentiable. Proceedings of the American Mathematical Society, 133, 2005, pp. 859-863.
[6] R.F. CURTAIN and H.J. ZWART, An Introduction to InfiniteDimensional Linear Systems Theory, Springer, New York, 1995.

[7] P. Destuynder, I. Legrain, L. CASTEL and N. Richard, Theoretical, numerical and experimental discussion on the use of piezoelectric devices for control-structure interaction, Europ. J. Mecha. A/Solids, 11, 1992, pp. 181-213.

[8] C. DE Boor, A pratical quide to spline, In Applied Mathematical Sciences Vol.27, Springer, 1978.

[9] A. De LuCA AND B. Siciliano, Trajectory control of a non-linear one-link flexible arm, Int. J. Control. 50, 1989, pp. 1699-1715.

[10] V. DitKine and A. ProudniKov, Transformations intégrales et calcul opérationnel (traduit du russe), Mir, Moscou, 1978.

[11] D. EISEnBUd, Commutative Algebra with a View Toward Algebraic Geometry, Springer, New York, 1995.

[12] A. El JAI and A.J. PRITCHARD, Capteurs et actionneurs dans l'analyse des systèmes distribués, Masson, Paris, 1986.

[13] A. FABIASKA and A. QuAdrat, Applications of the Quillen-Suslin theorem to multidimensional systems theory, in Grbner Bases in Control Theory and Signal Processing, H. Park, G. Regensburger (Eds.), Radon Series on Computation and Applied Mathematics, de Gruyter publisher, 2007, pp. 23-106.

[14] N.FAiz, S. Agrawal and R. Murray, Differentially Flat Systems with Inequality Constraints: An Approach to Real-Time Feasible Trajectory Generation, J. Guidance, Control, and Dynamics, 24, 2001, pp. 219 227.

[15] T. Faulwasser, V. Hagenmeyer and R. Findeisen, Optimal Exact Path-Following for Constrained Differentially Flat Systems, Proc. of 18th IFAC World Congress, Milano, Italy, 2011, pp. 9875-9880.

[16] M. FLIESS, Some basic structural properties of generalized linear systems, Systems Control. Lett., 15, 1990, pp. 391-396.

[17] M. Fliess, J. Lévine, P. MARtin and P. Rouchon, Flatness and defect of non-linear systems: introductory theory and applications, Internat. J. Control, 61, 1995, pp. 1327-1361.

[18] M. Fliess and H. MounIER, Quelques propriétés structurelles des systèmes linéaires à retards constants, C.R. Acad. Sci. Paris, I-319, 1994, pp. 289-294.

[19] M. FLIESS and H. MouniER, Interpretation and comparison of various types of delay system controllabilities, Proc. IFAC Conf. System Struct. Control, Nantes, 1995, pp. 330-335.

[20] M. FLIESS and H. MoUNIER, Controllability and observability of linear delay systems : an algebraic approach, ESAIM Control Optim. Calc. Var., 3, 1998, pp. 301314.

[21] M. Fliess, H. Mounier, P. Rouchon and J. RudolPh, Controllability and motion planning for linear delay systems with an application to a flexible rod, Proc. $34^{\text {th }}$ Conf. Decision Control, New Orleans, 1995, pp. 2046-2051.

[22] H. GLÜSING-LÜERSSEN, A behavioral approach to delay-differential systems. SIAM J. Control Optim. 35(2), 1997, pp. 480-499.

[23] I.M. Guelfand AND G.E. Chilov, Generalized functions; vol. 2: fundamental spaces (translated from russian), Academic Press, New York, 1968.

[24] L. Hormander, The Analysis of Linear Partial Differential Operators II: Differential Operators with Constant Coefficients, Springer-Verlag, Berlin, Heidelberg, New York, 1990.

[25] H. Komatsu, Ultradistributions. I. Structure theorems and a characterization, J. Fac. Sci. Univ. Tokyo Sect. IA Math., 20, pp. 25105, 1973.

[26] H. Komatsu, Ultradistributions. II. The kernel theorem and ultradistributions with support in a manifold, J. Fac. Sci. Univ. Tokyo Sect. IA Math., 24, pp. 607-628, 1977.

[27] V. Komornik, Exact Controllability and Stabilization, Wiley, Chichester \& Masson, Paris, 1994.

[28] J.E. Lagnese and J.-L. Lions, Modelling Analysis and Control of Thin Plates, Masson, Paris, 1988.

[29] S. LANG, Algebra, $3^{\text {rd }}$ ed., Addison-Wesley, Reading, MA, 1993.

[30] H. LAOUSY, C.Z. XU and G. SALlET, Boundary feedback stabilization of a rotating body-beam system, IEEE Trans. Automat. Control, 41, 1996.

[31] B. Laroche, P. Martin and P. Rouchon, Motion planning for a class of partial differential equations with boundary control, In Proc. of Control and Decision Conference, Tampa, 1998.

[32] E. K.LAVROSSKY AND FORMAL'SKY, On the stabilization of the angular position of an elastic rod. Technical Cyberneticss, 6, 1989, pp. 115-123 (in Russian). 
[33] J.-L. Lions, Contrôle optimal des systèmes gouvernés par des équations aux dérivées partielles, Dunod, Paris, 1968.

[34] J.-L. Lions, Contrôlabilité exacte, perturbations et stabilisation des systèmes distribués, vol. 1 \& 2, Masson, Paris, 1988.

[35] A.F. LYNCH and J. RUDOLPH, Flatness-based boundary control of a class of quasilinear parabolic distributed parameter systems, International Journal of Control, 75(15), 2002, pp. 1219-1230.

[36] O. MoRGÜL, Dynamic boundary control of a Euler-Bernoulli beam, IEEE Trans. Automat. Control, 37, 1992, pp. 639-652.

[37] O. MoRgüL, Control and stabilization of a rotating flexible structure, Automatica, 30, 1994, pp. 351-356.

[38] H. MOUNIER, An algebraic interpretation of the spectral controllability of a linear delay system, Proc. $3^{\text {rd }}$ Europ. Control Conf., Rome, 1995, pp. 3325-3329.

[39] H. MounIER, Propriétés structurelles des systèmes linéaires à retards : aspects théoriques et pratiques, Thèse, Université Paris-Sud, Orsay, 1995.

[40] H. MounIER, Algebraic interpretations of the spectral controllability of a linear delay system, Forum Math., to appear.

[41] H. Mounier, J. Rudolph, M. Petitot and M. Fliess, A flexible rod as a linear delay system, Proc. $3^{\text {rd }}$ Europ. Control Conf., Rome, 1995, pp. 3676-3681.

[42] H. Mounier, J. Rudolph and F. Woittennek, Boundary value problems and convolutional systems over rings of ultradistributions., Advances in the Th. of Contr., Sig. and Sys. with Physical Modeling, vol. 407 of Lect. Notes in Control and Information Sciences, SpringerVerlag, Berlin, 2010, pp. 179-188.

[43] J. OldEnburg and W. MARQuARDT, Flatness and higher order differential model representations in dynamic optimization, Comp. \& Chem. Eng., 26, 2002, pp. 385-400.

[44] N. Petit, M. Milam, and R. Murray, Inversion based constrained trajectory optimization, In Proc. of 5th IFAC symposium on nonlinear control systems, 2001.

[45] A. QUADRAT, An introduction to constructive algebraic analysis and its applications, les cours du CIRM, 1 no. 2: Journes Nationales de Calcul Formel, 2010, pp. 281-471.

[46] D. QuILlen, Projective modules over polynomial rings, Inventiones Math., 36, 1976, pp. 167-171.

[47] J.-P. Ramis, Dévissage Gevrey, Astérisque, 59-60, 1979, pp. 173-204.

[48] J.-P. RAmis, Séries divergentes et théories asymptotiques, Soc. Math. France, Marseille, 1993.

[49] L. Rodino, Linear Partial Differential Operators in Gevrey Spaces, Singapore: World Scientific, 1993.

[50] J. Rotman, An Introduction to Homological Algebra, Springer-Verlag, New York, 2009.

[51] L.H. Rowen, Ring Theory, Academic Press, Boston, 1991.

[52] J. RudolPh, Duality in Time-Varying Linear Systems: A Module Theoretic Approach, Linear Algebra Applications, to appear, 1996.

[53] J. Rudolph and F. WoittenneK, Motion planning and open loop control design for linear distributed parameter systems with lumped controls, International Journal of Control Vol.81, 2008, pp. 457-474.

[54] P. SchapIRA, Microdifferential Systems in the Complex Domain, Springer, Berlin, 1985.

[55] H. Sira-RamíreZ, and S. Agrawal, Differentially flat systems, Control Engineering Series, Marcel Dekker, New York, 2004.

[56] A.A. SUSLIN, Projectives modules over a polynomial ring are free (in russian), Dokl. Akad. Nauk. SSSR, 229, 1976, pp. 1063-1066 (english translation: Soviet. Math. Dokl. 17, 1976, pp. 1160-1164).

[57] M. TUCSNAK, Regularity and exact controllability for a beam with piezoelectric actuator, SIAM J. Control Optimiz., 34, 1996, pp. 922-930.

[58] F. WoittenneK and $\mathrm{H}$. Mounier, Controllability of networks of spatially one-dimensional second order p.d.e. - an algebraic approach., Siam J. Contr., vol. 48, 2010, pp. 3882-3902.

[59] C.Z. XU and J. BAILlieUL, Stabilizability and stabilization of a rotating body-beam system with a torque control, IEEE Trans. Automat. Control, 38, 1993, pp. 1754-1765. 